\title{
Melanocytes from the Outer Root Sheath of Hair Follicles Cultivated on Collagen Membrane Improve their Melanotic Properties
}

\author{
Vuk Savkovic $^{1 *}$, Katharina Sülflow ${ }^{1}$, Katrin Rabe ${ }^{1}$, Marie Schneider ${ }^{1}$ and Jan-Christoph Simon ${ }^{2}$
}

${ }^{1}$ Translational Centre for Regenerative Medicine, Leipzig University, Germany

${ }^{2}$ Department of Dermatology, Venereology and Allergology, Leipzig University Medical Center, Leipzig, Germany

\begin{abstract}
Local depigmentation disorders such as Vitiligo are detrimental for both physiological and psychological reasons and demand a causal, non-invasive treatment. The only available treatment of a kind at present is melanocyte transplantation, disadvantageously dependent on an invasive skin biopsy as melanocyte source. We have previously reported our work on an autologous, non-invasive method of differentiating melanocytes from the outer root sheath of human hair follicles for use in transplantation treatment. Choice of a suitable biocompatible, biodegradable carrier as a temporary niche for transplanted melanocytes can be crucial in such applications. Therefore, we tested decellularized bovine collagen type I scaffold membranes as a candidate for a favourable carrier of the hair follicle melanocytes.

This study provides an additional insight to the reported upgraded explant method of cultivating melanocytes from human hair follicle and the advantages of collagen I membrane as a biocompatible carrier for them. We have displayed detailed analysis and detailed interpretation of the methodological improvements, showing that the upgraded explant procedure favoured the release, migration and proliferation of cell content with more cultivating potential and manifold higher melanocyte yield. The three-dimensional culture of melanocytes, seeded on collagen I membranes, displayed regular melanocyte markers, proliferated more rapidly and produced more melanin than the two-dimensional adherent culture. We concluded that the collagen I decellularized scaffolds provided a benevolent niche to hair follicle melanocytes and even upgraded their melanotic properties, which makes collagen I membranes an excellent candidate for a biocompatible carrier in future clinical applications.
\end{abstract}

Keywords: Outer root sheath; Hair follicle; Melanocyte; Vitiligo; Stem cells; Transplantation; Collagen I

\section{Introduction}

\section{Vitiligo}

Vitiligo is a skin disorder manifested as local depigmentation. Depigmented spots (so called white patches) severely lack functional melanocytes in the epidermis and therefore remain deprived of melanin, the pigment necessary for protection against solar radiation. The skin of the white spots becomes extremely sensitive to the sun and burns strikingly easily, having no innate protection. Perhaps even more sinister, white patches are often regarded as disfigurement, more than ever in the times of high cosmetic standards, especially if the patches are situated on exposed parts of the body such as face, arms and hands (38\% of depigmented surfaces in Vitiligo patients) [1]. All of the mentioned has a tremendous impact on life quality and social life of the affected (52\% single patients, [1-7]) and can lead to psychiatric morbidity in $34 \%$ of Vitiligo patients $[1,2,8]$, and devastating chronic depression (24\%) [1]. The urge to treat Vitiligo is strong in times of intensifying cosmetic awareness.

\section{Therapeutic principles}

The existing conservative therapies such as phototherapy, topical immune suppressors or chemical depigmentation are alleviating, short-term and non-causal, which imposes an unmet need of a causal Vitiligo treatment with a long-lasting effect [9-11].

\section{Transplantation therapy}

The closest approach to the causal, in terms of addressing the problem of melanocyte loss/absence, is delivering functional melanocytes to the points of demand (i.e., melanocyte transplantation). Melanocyte transplantation has been used as a standard procedure for quite long as reported by several groups [12-18]. For all of the transplantation procedures, melanocytes are harvested from the epidermis upon excision of a piece of skin, differing in the ways of handling the cells after the biopsy. The simplest procedure involved resuspension of the cells from digested epidermis and subsequent application of the entire cell suspension onto previously dermabraded patches [18]. The heterogeneous content of such suspensions has an advantageous ratio of epidermal cells, especially that of keratinocytes vs. melanocytes, but also carries an innate peril of non-sterile or in best case semi-sterile isolation procedure, resulting in roughly one third of graft rejections due to mycosis [19]. Further on, the melanocytes can be isolated and grafted or further cultivated [19], proliferated and grafted. Some of the performed melanocyte transplantations have brought about pigmentation improvement on the depigmented skin patches [12-19]. Nevertheless, all of the procedures involved a surgical removal of up to $6 \mathrm{~cm}^{2}$ of skin.

*Corresponding author: Vuk Savkovic, Translational Centre for Regenerative Medicine, Leipzig University, Phillip-Rosenthal-Str. 55, 04103 Leipzig, Germany, Tel: +49 341 9739647; Fax: +49 341 9739609; E-mail: vsavkovic@trm.uni-leipzig.de

Received November 19, 2012; Accepted December 19, 2012; Published December 21, 2012

Citation: Savkovic V, Sülflow K, Rabe K, Schneider M, Simon JC (2012) Melanocytes from the Outer Root Sheath of Hair Follicles Cultivated on Collagen Membrane Improve their Melanotic Properties. J Tissue Sci Eng S11:004. doi:10.4172/2157-7552.S11-004

Copyright: ( 2012 Savkovic V, et al. This is an open-access article distributed under the terms of the Creative Commons Attribution License, which permits unrestricted use, distribution, and reproduction in any medium, provided the original author and source are credited. 


\section{Non-invasive procedures}

In times of a strong and persistent trend towards non-invasive or minimally invasive procedures, it is very important that material sampling remains non-invasive. This aspect involves even more sensitivity when it comes to treatment of skin disorders due to a visible cosmetic result of the application. Therefore, difference between sampling and biopsy carries much significance when the starting material for the cell therapy is being harvested. In that sense, sampling hairs with follicles by plucking (completely painless and harmless procedure finalised in matter of minutes) presents us with a noninvasive replacement for an invasive excision biopsy of skin (procedure which involves pain, bleeding, requires post-operative care and leaves a scar).

The melanocytes cultivated from the Outer Root Sheath (ORS) of hair follicles provide a possibility of transplantation onto the prepared depigmented patches of skin. Several application routes are possible in terms of transplantation. The simplest procedure is by all means an application of cell suspension, liquid or in the form of aerosol, stabilised by fibrin and/or thrombin, which works well on even surfaces. Pioneer study of Vanscheidt and Hunziker [20] involved successful transplantation of a whole ORS suspension onto a Vitiligo patch, without prior isolation of melanocytes. Further application variants involve biocompatible carriers that mechanically and biologically stabilize the HM before, in the course of transplantation and posttransplantationally. Candidates for such carriers are of cellular nature (for instance, keratinocyte-based epidermal equivalents), scaffolds based on cellular derivates (such as collagen-based decellularized scaffolds) or scaffolds synthesized from synthetic biocompatible materials. Modifications of the carriers involve a remarkably high number of variants.

\section{Goals}

We have initially used the experimental platform of Dieckman et al. [21] to establish a medium-air-interface culture of hair follicles and subsequent adherent culture of ORS melanocytes. Despite the reported high purity, the cultivation timeline of two months left room for more efficiency. Along the way, we introduced several improvements to the initial Dieckmann experimental procedure [22].

In this article, we have provided further analysis of the upgraded method and of the cells obtained. We have additionally compared the two methods, Dieckmann explant method [21] and the upgraded method [22] at each phase of the culture, gaining further data that not only explained the dynamics and differences in the early stages, but also revealed significant accumulative differences in cell numbers at the advanced stages of the culture.

Using the human HM cells obtained from the upgraded method, we have tested decellularized bovine collagen type I-based membranes (Collagen Cell Carrier, CCC, [23]) as biocompatible carriers for HM that were cultivated in improved conditions described in [22] and reflected in this study.

\section{Material and Methods}

\section{Cultivation, expansion, differentiation and selection of pure melanocyte culture}

Human outer root sheath melanocytes (HM) were cultivated from the ORS of human follicles as described [22]. Briefly, the follicles were shortened at the proximal end, partially digested with collagenase $\mathrm{V}$ and cultivated in medium-air-interface conditions until the stem cells and progenitor cells migrated out of the follicle and the progenitors proliferated on a nylon mesh. After harvesting the cells by trypsinization, adherent culture was performed until the cell showed correct melanocyte morphology, expressed Tyrosinase, glycoprotein 100 (gp100) variants and produced melanin using a set of enzymes (Tyrosinase, Tyrosinase-Related Protein 1 and 2). The ORS melanocytes were selected during the adherent culture by the means of differential trypsinization and Geneticine selection, which helped withdraw keratinocytes and fibroblasts as usual contaminants of the ORS melanocyte culture. Basic steps of the method are shown in figure 1. Since the combined improvements of the upgraded method yielded one million pure functional melanocytes in four weeks instead of the initial eight weeks [22], we analyzed the features of all cultivation steps of the explant culture. We performed comparison experiments of the initial (Dieckmann) versus the upgraded explant method, monitoring follicle activity, follicle surface, cell numbers, outcome of Geneticine selection, marker expression and total cell yield.

For further comparison purposes, we performed a parallel cultivation procedure of intact hair follicles, cultivated in medium-airinterface with supplemented M2 Promocell medium [24], as described in Dieckmann et al. [21], with and without Geneticine selection step during adherent culture described in our upgraded procedure [22]. The initial method of selective elimination by Geneticine can be found in [25].

\section{Follicle activity}

The follicles cultivated in medium-air-interface, according to both the method of Dieckmann and the current method, were monitored for growth activity and regularly documented (Figure 2).

\section{Measurement of the outer root sheath surface changes}

Surface of the follicle ORS was measured by the means of Nikon NIS Elements BR (Version: 3.00) software. The ORS surface, which is morphologically clearly distinguishable, was selected from surface micrograph of hair follicle by the means of an adjustable polygonal frame and the surface values were read out by software. The average values of ORS surface of four independently set up cultivation experiments with five technical replicates were compared at day 0 , day 7 and day 14 (Figures 3 and 4).

\section{Cell numbers}

After harvesting the cells released from the ORS in the course of medium-air-interface cultivation, the cells were further cultivated
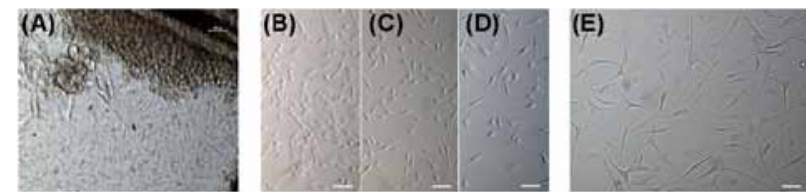

(A) Shortened, active follicle pre-treated with collagenase $V$ and cultivated in medium-air-interface conditions with migrating cells on the surrounding mesh forming own colonies. (B) Non-selected cells in the adherent culture (C) Geneticine challenged cells in the adherent culture (D) differentiated ORS melanocytes (HM) (E) Control: Normal Human Epidermal Melanocytes (NHEM).

Figure 1: Current method for cultivating melanocytes from the outer root sheath cell pool of the hair follicle according to Savkovic et al. [22]. 
Citation: Savkovic V, Sülflow K, Rabe K, Schneider M, Simon JC (2012) Melanocytes from the Outer Root Sheath of Hair Follicles Cultivated on Collagen Membrane Improve their Melanotic Properties. J Tissue Sci Eng S11:004. doi:10.4172/2157-7552.S11-004

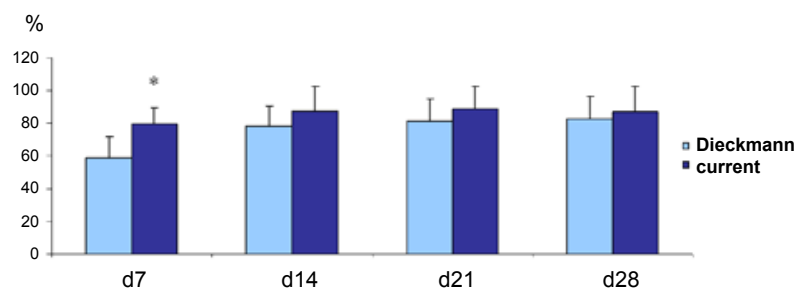

Both the current method and the method of Dieckmann et al. [21] comprised an active outgrowth of hair follicle outer root sheath. The percentage of active follicles cultivated according to the current method was higher than that of follicles cultivated according to the Dieckmann method, as measured at day 7 , 14,21 and 28 . At day 7, the difference in percentage of active follicles between the two methods was statistically significant $\left(p=0.038^{*}\right)$, whereas on day 14 , 21 and 28, the trend persisted on a non-significant level $(p>0.05)$.

Figure 2: Comparative changes in follicle activity.

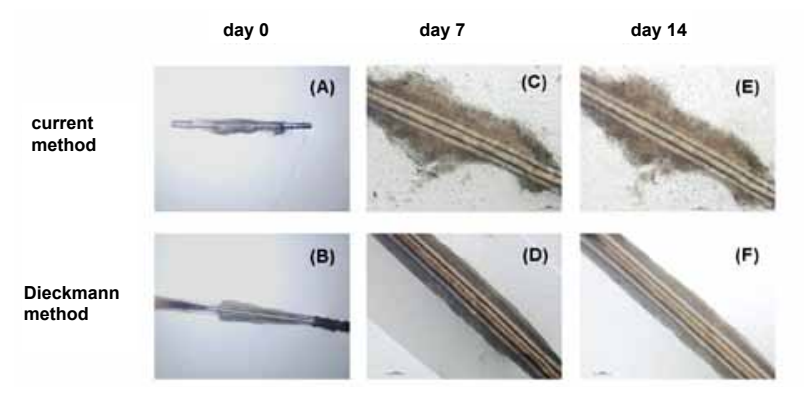

Example of hair follicles cultivated in the medium-air-interface upon the current method (A, C, E) displaying higher increase in ORS surface than those cultivated according to the Dieckmann method (B, D, F). On day 0 , all of the follicles were compact $(A, B)$. On day 7 , outgrowth and migration of the cells was evident $(C, D)$, as well as on day $14(E, F)$. Stronger outgrowth of the follicles cultivated according to the current method was visible (C, E). (A) Current method, day 0; (B) Dieckmann method, day 0; (C) Current method, day 7; (D) Dieckmann method, day 7; (E) Current method, day 14; (F) Dieckmann method, day 14.

Figure 3: Representative photographs of comparative ORS surface changes.

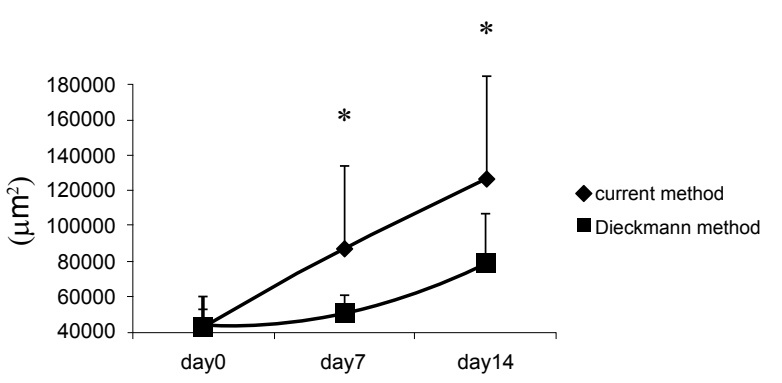

Both the current method $(\bullet)$ and the method of Dieckmann et al. ( $\bullet$ ) comprised an increase of the ORS surface. The surface increase was significantly higher in ORS of the follicles cultivated according to the current protocol in comparison with that of the Dieckmann method, as measured at day 7 (ORS surface ratio $0.69, p=0.01^{* *}$ ) and day 14 on day 14 (ORS surface ratio 0.72 , $\left.p=0.018^{*}\right)$.

Figure 4: Comparative changes of the Outer Root Sheath surface.

according to the specifications of both methods. The cells were stained with Trypan Blue and counted in Neubauer chamber at each passage in order to determine the total cell number in the culture. The passage at the day of harvesting the cells from the medium-air-interface and their set-up as adherent culture was nominated passage 0 (Figures 5 and 6).

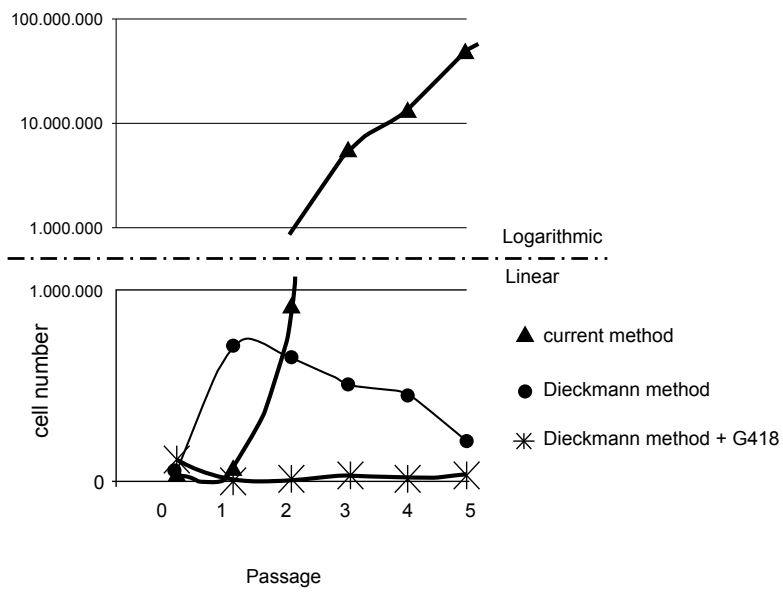

Example of growth curves of ORS cells cultivated according to the current method $(\boldsymbol{\Delta})$, Dieckmann explant method $(\bullet)$, Dieckmann explant method with $50 \mu \mathrm{g} / \mathrm{ml}$ Geneticine supplement over $48 \mathrm{~h}$ at early passages of adherent culture $\left({ }^{*}\right)$. The lower part of the graph is displayed linearly and the upper part logarithmically. The cells cultivated upon the method described herein reached the number of $1,000,000$ cells by the second passage and as much as $80,000,000$ cells by the passage 5 of adherent culture (displayed results of single donor using comparative methods).

Figure 5: Comparative cell growth in the adherent culture.

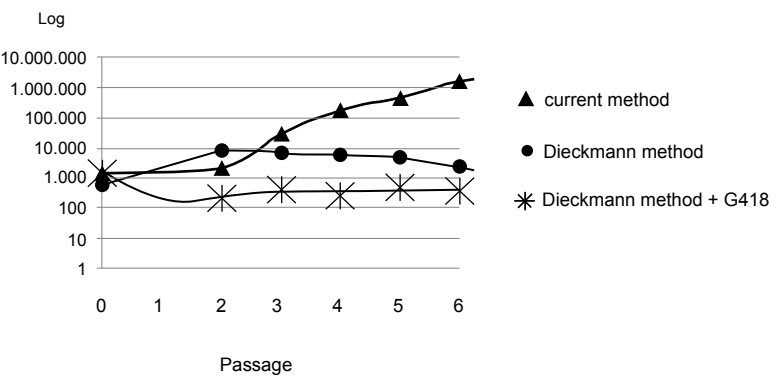

Growth curves of ORS cells per sampled hair follicle cultivated according to the current method $(\boldsymbol{\Delta})$, Dieckmann explant method $(\bullet)$, Dieckmann explant method with $50 \mu \mathrm{g} / \mathrm{ml}$ Geneticine supplement over $48 \mathrm{~h}$ at early passages of adherent culture $\left(^{*}\right)$. The graph is displayed logarithmically. The theoretical average cumulative cultivation potential per single follicle reached as much as 2,700,000 melanocytes.

Figure 6: Comparative growth in the adherent culture per hair follicle.

\section{Comparison of the Dieckmann method and herein provided method with geneticine addition}

Additionally, in order to withdraw the contaminant fibroblasts and keratinocytes as competitors against differentiating melanocytes, we have treated the 'Dieckmann' adherent culture with $50 \mu \mathrm{g} / \mathrm{ml}$ geneticine after the $\mathrm{p} 0$ as a corresponding selection measure used in the current method. Geneticine affects translation and hereby the quicklydividing cells with high demand for protein synthesis, therefore the melanocytes survive Geneticine treatment, whereas the fibroblasts and keratinocytes do not $[22,25,26]$.

This part of the treatment was also intended to clarify the effect of Geneticine on the amplified heterogeneous cell pool harvested according to the Dieckmann et al. [21], e.g. to estimate the cellular composition of such cell pool. Working hypothesis predicted a small 
Citation: Savkovic V, Sülflow K, Rabe K, Schneider M, Simon JC (2012) Melanocytes from the Outer Root Sheath of Hair Follicles Cultivated on Collagen Membrane Improve their Melanotic Properties. J Tissue Sci Eng S11:004. doi:10.4172/2157-7552.S11-004

number of survivor cells in case of the high number of contaminant fibroblasts and keratinocytes and vice versa-more survivors of the Geneticine treatment in case of the low contaminant cell content (Figures 5 and 6).

\section{Cultivation of HM cells on collagen membranes}

Bovine decellularized collagen I membrane scaffolds, also called CCC (Collagen Cell Carrier, Viscofan Bioengineering, Weinheim, Germany) were tested as a temporary niche for ORS melanocytes for purposes of grafting.

The scaffolds were circle-shaped with a sterile hollow punch, with an $8 \mathrm{~mm}$ diameter so that they covered the $10.7 \mathrm{~mm}$ wide bottom of the $360 \mathrm{~mm}^{2}$ wells (96-well-plate) in calibrated condition. The membranes were placed on the bottom of the 96-well and calibrated with sterile PBS and subsequently with DLM medium for 24 hours. The position of the membranes and presence of air bubbles were inspected and they were tightly positioned to the bottom of the wells using sterile forceps. The medium used for calibration was discarded. Resuspended cells were seeded over the membranes and left to adhere, 10,000 cells per scaffold, resuspended in $200 \mu$ medium.

Over the next 5 days, the HM cells were populating the collagen membrane and proliferating (Figure 7).

\section{Immunofluorescence}

The cells on scaffolds were fixed with paraformaldehyde (PFA) and labelled with Tyrosinase and NKI-beteb mouse-generated antibodies, followed by the secondary labelling by an Alexa 647 anti-mouse antibody as described [22]. The cells on scaffolds were washed with PBS and fixed with ice-cold Para formaldehyde (PFA, 4\%) for 8 minutes, then washed with PBS again. The cells were blocked with $0.5 \mathrm{ml}$ of $2 \%$ bovine serum albumin $2 \%$ BSA-PBS and permeabilised with $0.1 \%$ Triton PBS solution for $1 \mathrm{~h}$. Solutions of primary mouse-generated antibodies against NKI/beteb and Tyrosinase were set at $2 \%$ in PBS with $2 \% \mathrm{BSA} / 0.1 \%$ Triton and incubated with the cells on scaffolds overnight at $4^{\circ} \mathrm{C}$. After washing with $2 \% \mathrm{BSA} / 0.1 \%$ Triton solution, biotinylated anti-mouse secondary antibody was added to the cells

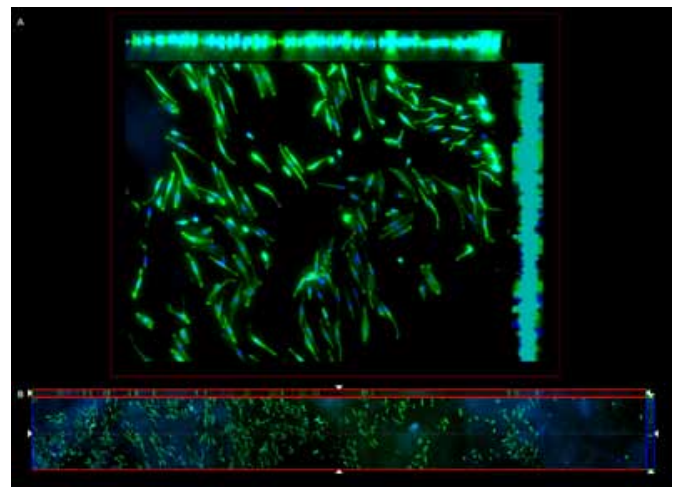

(A) Maximum Intensity Projection (MPI) z-stacked take of $\mathrm{HM}$ on CCC membrane. Side projections display the depth of the scaffold reconstructed from 10 consecutive images taken with a $10 \mu \mathrm{m}$ progression interval. The cells populated the $100 \mu \mathrm{m}$ depth of the CCC scaffold. Over $97 \%$ of the cells expressed, the gp100 variant NKI-beteb, present in melanosomes of ORS melanocytes (HM). (B) 'cross-census' panoramic image of 7 images consecutively taken along the same axis across the full width of a mounted slide.

Figure 7: HM cells cultivated on bovine collagen membrane (CCC). and incubated for $1 \mathrm{~h}$, then washed. Solution of $2 \%$ BSA-PBS/0.1\% Triton/0.25\% IGG was incubated with the cells for one hour, and then washed. Finally, Streptavidin-coupled Alexa594 ${ }^{\circledR}$ (red) and Alexa $488^{\circledR}$ (green) fluorochrome $0.25 \%$ solution in $2 \% \mathrm{BSA} / 0.1 \%$. Triton were incubated with the cells together with $1 / 1000$ DAPI nuclear dye, for $1 \mathrm{~h}$, then washed two times with $2 \%$ BSA/0.1\% Triton solution. Scaffolds with the cells were left to dry out for 2 minutes, fixed onto glass with Fluoromount ${ }^{\circledR}$ sealing liquid, and covered with a glass slip.

\section{Microscopic analysis of the 3D HM cultures}

The marker signals were analysed by the means of fluorescence microscopy (Nikon Eclipse Ti-S; Nikon Corporation, Deutschland) and ApoTome Laser-Scanning microscope (LSM 510 Meta, Carl Zeiss Jena GmbH, Germany).

Reconstruction of the three-dimensional structure of the membraneadherent HM culture was analyzed by the means of ' $z$-stacking'. Images of the HM cells seeded onto the decellularized bovine collagen I membranes were taken using the ApoTome microscope system (Zeiss) navigated by AxioVision Software. Serial images were taken along the $\mathrm{z}$-axis at different depths of the $100 \mu \mathrm{m}$ membrane with a progressing interval of circa $10 \mu \mathrm{m}$ (z-stack function). The resulting 10 images were integrated into a single Maximum Intensity Projection (MIP) image. The incorporated visual information of the MIP images enabled an estimate of the depth along the $\mathrm{z}$-axis and modelling of the threedimensional structure (Figure 7).

For the purposes of analysis and in order to avoid inconsistencies in choice of a random field for analysis, seven $\mathrm{z}$-stacks in a row were taken along the horizontal axis of the slide and built into a single panoramic image using MosaiX-function of the AxioVision Software, creating a cross-census of each mounted slide. The images were analyzed by the means of public domain ImageJ 1.42q Software [27]. Tyrosinase and NKI-beteb signals were analyzed along with the nuclear DAPI-signals to derive the expression index.

\section{Mitochondrial activity}

Viability and further on proliferation of the HM cells were monitored by the means of tetrazolium-reduction-based WST test (Roche Applied Science, Germany) after 5 days as described in [28]. WST dye was diluted 1:10 in DMEM medium. The medium above adherent cells and scaffolds was aspirated. The cultures were washed once with PBS buffer, $100 \mu \mathrm{l}$ of diluted WST-dye was added over the cells and left at $37^{\circ} \mathrm{C}$ to incubate. After the incubation time of 120 minutes, the conditioned WST-reagent mixture was transferred into empty 96 -wells and the extinction at $450 \mathrm{~nm}$ was measured by the means of Multiscan ${ }^{\circledR}$ Spectrum Photometer (Thermo Fisher Scientific, Germany). The background extinction values of blank wells filled with DMEM were subtracted from the values of the conditioned WST reagent mixture.

The WST values measured during the five-day-culture were compared to the cell counts determined by Trypan Blue exclusion and counted by the means of Neubauer chamber (Figure 8).

\section{Melanin production}

Melanin content was quantified by complete lysis of cell structures, including melanosomes and subsequent measurement of lysate extinction, as described [22]. 


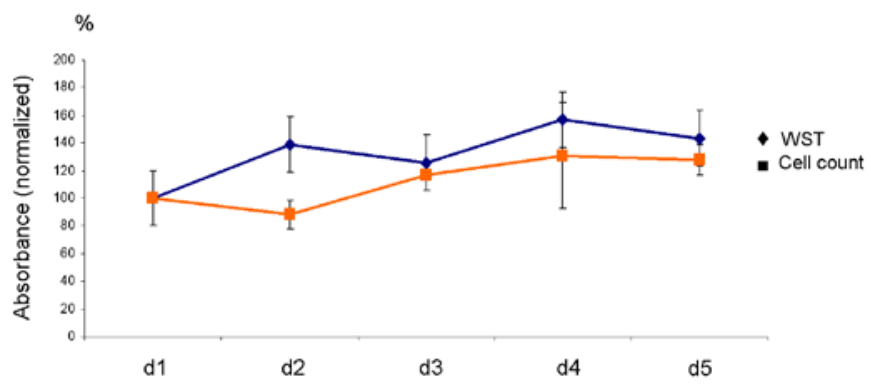

The seeded $10,000 \mathrm{HM}$ cells proliferate during 5 days of cultivation, reaching 12,500 cells. The increase of $27.5 \%$ corresponds to the increase of $43 \%$ in the mitochondrial activity measured at day 5 .

Figure 8: Mitochondrial activity increase over 5 cultivation days in adherent culture corresponded to the increase in cell count.

\section{Statistical analysis}

All the experiments were performed with material of four donors and performed in biological triplicates with five technical replicates. The statistical analysis was performed by the means of a two-tailed $\mathrm{t}$-test, with significance threshold set at $0.05 \mathrm{p}$ value $\left(\mathrm{p}<0.05^{\star}\right.$ for significant, $\mathrm{p}<0.01^{* *}$ for highly significant, $\mathrm{p}<0.001^{\star * *}$ for very highly significant). All the $\mathrm{p}$ values higher than $\mathrm{P}=0.05$ were regarded as nonsignificant.

\section{Results}

\section{Cultivation, expansion, differentiation and selection of pure} melanocyte culture

The upgraded procedure has yielded a higher-expanded culture of 95-100\% pure, almost fully differentiated human melanocytes, which reached the melanotic stage within 3 to 4 weeks post-hair plucking, as early as p3 or p4 of the primary culture [22]. In particular, the cultivation and differentiating procedure from 60 hair follicles yielded $10^{6}$ pure, differentiated human melanocytes in melanotic stage within 3-4 weeks post-epilation, in the second to fifth passage of adherent culture. In comparison to the results of Dieckman et al. [21], the method presented here yielded an output of $10^{6} \mathrm{HM}$ in half the time and by far exceeded the yield of protocol by Dieckmann already in very early passages of adherent culture according to our findings.

Furthermore, the improved procedure reached much higher numbers in the further passages of the adherent culture ( $p 3$ to p6).

\section{Morphological characteristics}

HM and NHEM were morphologically quite similar. Both cell types have a typically large soma that can vary in surface. The soma of HM tends to be somewhat smaller than that of NHEM. Both cell types developed two or more (typically 3-4) dendrites. Both HM and NHEM produced vesicles with melanin-melanosomes transported from the soma towards dendrite tips, visible as dark inclusions (Figure 1).

\section{Follicle activity}

Number of follicles that yielded migrating cells at mediumair-interface cultivating system was higher in follicles pre-treated according to the current method (discarding of the proximal part and digesting with collagenase V). At day 7 of the follicle culture, $79.75 \%$ of the follicles in our culture were outgrown in comparison to the $58.81 \%$ of intact follicles cultivated upon Dieckmann et al. [21] $\left(\mathrm{p}=0.038^{\star}\right)$. In the later stages, this difference persisted on a non-significant level ( $>0.05$, Figure 2).

\section{ORS surface growth}

Difference in growth between methods was visible already after day 7 of the medium air interface follicle cultivation on nylon meshes, with clearly displayed enhanced growth of the follicles cultivated upon the method described herein in comparison to the Dieckmann method (Figure 3). The ORS surface of hair follicles digested with collagenase and cultivated according to the current method, increased from 42.480 $\pm 17.451 \mu \mathrm{m}^{2}$ on day 0 to $87.74 \pm 45.4 \mu \mathrm{m}^{2}$ on day 7 and $125.84 \pm$ $58.45 \mu \mathrm{m}^{2}$ on day 14 , whereas the follicles cultivated according to the Dieckmann method increased from $43.30 \pm 9.23 \mu \mathrm{m}^{2}$ on day 0 to 50.04 $\pm 10.75 \mu \mathrm{m}^{2}$ on day 7 and $77.49 \pm 29.57 \mu \mathrm{m}^{2}$ on day 14 (Figures 3 and 4). The ratio of the Outer Root Sheath surface between the Dieckmann method and the current method hereby declined from 0.94 on day 0 to 0.69 on day $7\left(\mathrm{p}=0.01^{\star *}\right)$ and 0.72 on day $14\left(\mathrm{p}=0.018^{\star}\right)$, displaying faster growth and migration of cells from shortened, collagenasetreated follicles (Figure 3). At the point of harvesting the cells from the Transwell ${ }^{\circledR}$ nylon meshes in order to take them into adherent culture, the number of harvested cells exceeded that of the Dieckmann method by factor 1.84 when all the sampled hair follicles were taken into account.

\section{Comparative growth in adherent culture}

The cells in the adherent culture, cultivated according to the method described herein, continued to proliferate and reached the numbers of $1,000,000$ at the passage 2, adherent culture day 11 and as much as $80,875,000$ at passage 6 , day 44 (Figure 5). This means that each follicle was able to yield as much as 30,666 melanocytes in 5 weeks and upto 2,695,833 melanocytes within 9 weeks (Figure 6). In contrast to our method, the cells in the adherent culture cultivated according to the Dieckmann et al. [21] protocol reached 710,000 cells at passage 1 of the adherent culture as the highest cell number and 650,000 cells by passage 2 at day 11 of the adherent culture (reaching theoretical values of 7,717 cells per follicle at p1 and 7,065 cells per follicle at p2) (Figures 5 and 6). Unlike the cells cultivated upon the method described herein, the cells cultivated according to the Dieckmann method decreased in numbers with further passages (Figures 5 and 6).

\section{Geneticine treatment}

Geneticine treatment very efficiently helped in removing keratinocytes and fibroblasts from the culture, leaving large numbers of melanocytes for further cultivation (Figures 1,5 and 6). Additional Geneticine treatment of the adherent Dieckmann culture as a corresponding selection measure used in our current method resulted with extremely low numbers of survivor cells $(<2 \%$ confluence) (Figures 5 and 6).

\section{D collagen I scaffold cultivation}

The HM cells seeded on bovine collagen I membranes populated the scaffold, adhering well, interfacing with the scaffold and migrating the depth of $100 \mu \mathrm{m}$. The cells expressed melanocyte markers Tyrosinase and gp100 variant NKI-beteb (Figure 7). 98.17\% of the seeded cells expressed Tyrosinase and $97.13 \%$ expressed NKI-beteb variant of gp 100 , which fully corresponded to the reported $96 \%$ of markerexpressing cells in the adherent culture ([22], Figure 7). 
Citation: Savkovic V, Sülflow K, Rabe K, Schneider M, Simon JC (2012) Melanocytes from the Outer Root Sheath of Hair Follicles Cultivated on Collagen Membrane Improve their Melanotic Properties. J Tissue Sci Eng S11:004. doi:10.4172/2157-7552.S11-004

\section{Mitochondrial activity}

The 10,000 seeded HM cells proliferated both on polystyrene and CCC membrane over 5 cultivation days. The comparison of percentual growth in the adherent culture of HM shows that the extinction values of the WST test, as a measure of mitochondrial activity, corresponds to cell counts determined by Trypan Blue exclusion in the course of the five-day culture (Figure 8). Mitochondrial activity of HM cells seeded on CCC membranes and measured on day 5 exceeded that of the polystyrene-adherent HM culture by $28 \%\left(\mathrm{p}=0.026^{*}\right)$ (Figure 9$)$.

\section{Melanin production}

Melanin content of the HM cells cultivated on CCC membranes exceeded that of the polystyrene-cultivated HM by $19 \%(1.62 \pm 0.37$ $\mathrm{ng} /$ cell on CCC membrane, $1.36 \pm 0.22 \mathrm{ng} /$ cell polystyrene-adherent $\left.\mathrm{HM}, \mathrm{p}=0.038^{\star}\right)$ (Figure 10).

\section{Discussion}

The goal of the upgraded explants method was to differentiate stem cells and progenitor cells from ORS of hair follicle and obtain high amounts of differentiated cells that would, both in morphology and function, represent a peer to epidermal melanocytes.

As already shown in [22], combined upgrades of the Dieckmann method [21] brought about exceedingly high improvements in terms of cell yield. Discarding of fibroblasts from the cut-off follicle proximal part, loosening of the extracellular matrix by collagenase, favourable cultivation conditions with a low-serum and non-toxic DermaLife medium, along with combined selection methods of isolating melanocytes by differential trypsinization and eliminating

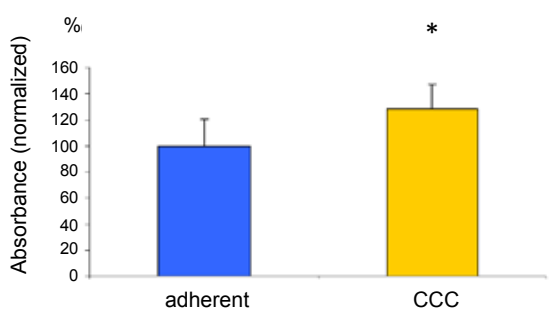

Proliferation of HM cells cultivated on CCC membranes, estimated by measuring the mitochondrial activity (WST test), exceeded that of polystyreneadherent $\mathrm{HM}$ by $28 \%\left(\mathrm{p}=0.026^{*}\right)$.

Figure 9: Comparative mitochondrial activity of polystyrene-adherent and CCC-cultivated HM culture.

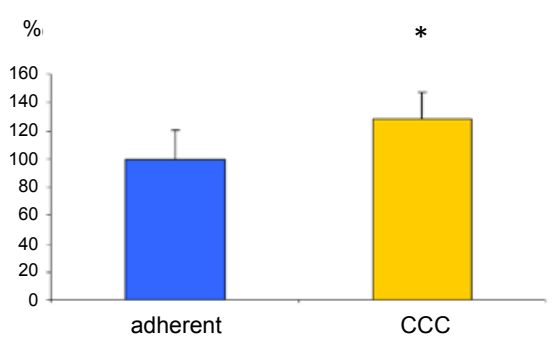

Melanin content of HM cells cultivated on CCC membranes, exceeded that of polystyrene-adherent HM by $19 \%\left(p=0.038^{*}\right)$.

Figure 10: Melanin content of the HM in adherent culture and 3D culture on CCC membrane. keratinocytes and fibroblasts by Geneticine challenge, resulted in a very reliable and reproducible method of cultivating melanocytes from the ORS. The upgraded method brought about not only one million of ORS melanocytes in four weeks, but also as much as 80 million cells within nine weeks, which represents a substantial cultivating potential.

\section{Follicle activity}

Outgrowth of stem cells and progenitors from the ORS during medium-air-interface cultivation was more rapid in our upgraded method than in those cultivated according to Dieckmann et al. [21]. For the purposes of this comparison, we have followed the number of outgrown follicles during medium-air-interface cultivation for 28 days (Figure 2). Whereas the difference between the current and Dieckmann method was more pronounced and statistically significant on day 7 of the follicle culture $\left(\mathrm{p}=0.038^{\star}\right)$, it decreased with time, upholding the trend on a non-significant level. Our interpretation of this decrease is that both methods involved follicle activation, but with qualitatively different cell content being released onto the nylon mesh. The Dieckmann method kept yielding substantial numbers of cells at this stage (visible as active follicles with cells leaving ORS), however those cells were prevalently keratinocytes and fibroblasts. This interpretation was corroborated by the low numbers of cells that remained after Geneticine selection of primary cell yield, which indicated that the melanocyte amount in the output adherent culture of the Dieckmann method was modest and by all means lower than according to the upgraded method (Figures 5 and 6).

\section{ORS surface changes}

Collagenase effect on loosening the extracellular matrix through collagen proteolysis had obviously enabled easier migration of the cells out of the ORS. This effect was visible by the enlarged surface of the ORS and by a more intensive migration of cells from ORS onto the nylon mesh (Figure 3). The initial growth of ORS in follicles digested with collagenase $\mathrm{V}$ as per the current method exceeded that of the non-digested follicles according to the Dieckmann method in all stages of adherent culture following day 0 (Figures 3 and 4). That gave the current method the advantage of harvesting more cells from the medium-air-interface follicle culture and having more cells for the set-up of the adherent culture to start with. The number of cells harvested from the follicles cultivated by the current method on day 0 of the adherent culture exceeded that of Dieckmann method by factor 1.84 (Figure 4).

\section{Morphology}

It is easy to comprehend that HM and NHEM are remarkably similar $[21,22]$. They have the same developmental origin $[21,22,29,30]$. ORS stem cells and progenitors regularly give rise to epidermal melanocytes that subsequently migrate out of hair follicle and home to the stratum basale for the purposes of epidermal regeneration [30-42].

Both HM and NHEM can be morphologically quite heterogeneous in appearance, depending on the time of adherence and passage.

HM and NHEM both displayed a typically large soma and several dendrites (in adherent culture, they are in average tripolar or tetrapolar) [21,22] (Figure 1). Cell size and number of dendrites often differed between donors and between passages in both NHEM and HM.

\section{Comparative growth in the adherent culture}

The cells in the adherent culture were continuously dividing in 
cultivation experiments according to the method described herein, reaching 1,000,000 cells at the passage 2 of the adherent culture, (half the time needed for the same number of cells cultivated according to Dieckmann et al. [21]) and as much as $80,875,000$ by the passage 6 (Figure 5). The cells cultivated according to the Dieckmann method did not reach the mentioned numbers; in fact, their numbers persistently declined after the passage 2 of the adherent culture.

The fact alone that a single hair follicle could in average produce almost 2.7 million cells in favourable culture conditions (Figure 6) speaks very much in favour of the ORS as a very promising source of stem cells and progenitor cells for regenerative therapies. As for the cultivation conditions, it is clear that the combined upgrades enabled quicker release and proliferation, and hereby accumulation of stem cells and progenitors at the early stages of cultivation before yielding them from the medium-air-interface cultivation set-up, which assured more cells for the adherent culture. Non-toxic and non-tumour-promoting medium DLM was evidently favourable enough to ensure further selection and amplification of melanocytes in the hypoxic culture. Elimination of fibroblasts and keratinocytes relieved the melanocyte culture of competitive pressure, which also helped the increase of the yield.

\section{Geneticine treatment}

Clearly, it was not only the number of cells, but also the cell content that proved favourable within the yield obtained from primary culture (medium-air-interface follicle cultivation). The portion of fibroblasts and keratinocytes in the material harvested from the medium-air-interface follicle primary culture was much higher in the preparation upon the Dieckmann method, judging by their massive cell death during and after the Geneticine selection. An extremely low number of cells that survived the Geneticine treatment indicated that the Dieckmann method did not provide enough stem cells and progenitor cells to quickly cultivate larger amounts of melanocytes in the adherent culture (Figures 5 and 6). In contrast to the outcome of Geneticine selection of the cells cultivated according to Dieckmann et al. [21], large number of cells cultivated as described in our upgraded method survived this selection step and those cells gave rise to very high amounts of melanocytes.

Altogether, the cells cultivated according to the current method reached higher numbers than the cells cultivated according to Dieckmann method, for several reasons. Upon the current method, the initial release and migration of stem cells and progenitor cells out of ORS onto the nylon mesh occurred in more follicles (Figure 2) and with higher intensity yielding more stem cells and progenitors from the primary culture to start with. Our interpretation of the primary culture yield includes a higher stem cell content vs. a lower fibroblast and keratinocyte content in the current preparation. This was deduced from the outcome of the subsequent selection steps (Geneticine challenge and regular differential trypsinization), which left much more living melanocytes behind than in the identical selection procedure of the adherent cells yielded from the primary culture according to the Dieckmann method (Figures 5 and 6).

Synergy of the higher start number of stem cells and progenitors in adherent culture and more favourable culture conditions given by the current method significantly advanced the total yield of HM.

\section{D membrane cultivation}

Main concerns by the choice of material for a graft scaffold is its biocompatibility, biodegradability and the kind of environment it can provide for the grafted cells. Collagen, being a major structural component of the body and present in the matrix of most natural tissues, has been widely utilized as a biocompatible, resorbable carrier material. Of all collagen types, collagen type I has been the most commonly used in regenerative medical applications, purified as well as additionally technologically manipulated into matrix variants with even higher biocompatibility. Xenogeneic (bovine or porcine) collagen I display low immunogenicity and can be applied in human tissues without risk of rejection [43]. Ectopic application of this primarily dermal protein appears to function without problems in skin grafting. Collagen type I as a physiological component of the skin is often used as a sole or combined component of dressings and biological membranes for wound healing $[44,45]$, as an epithelial graft $[46,47]$ and for delivery of epidermal or dermal cells [48]. As the most common protein of dermis, it has been plentifully used for dermal grafting [48], nevertheless it was just as efficient in epidermal grafting applications.

We have considered collagen type I as a carrier in melanocyte grafting applications for several reasons. General features of collagen I and its biocompatibility were certainly some of them. In addition, collagen I has an apparent temperate effect on melanocytes. Such favourable effects of collagen I coating as melanocyte niche have already been shown through disintegrating melanocytic spheroids and reconstituting morphological and physiological features of melanocytes [49]. This additional feature of collagen I is particularly worthy since melanocytes tend to be the limiting factor in co-cultured epidermal and dermal grafts and they are the first cell type to suffer loss [50]. Due to melanocyte sensitivity in grafts, providing a proper biological carrier niche is essential for grafting them. Moreover, collagen I has already been used as dressing and coating in melanocyte grafting applications, including Vitiligo treatment [51].

Enabling retainment of melanocyte dendritic morphology, melanotic function and migratory capabilities, along with biodegradability and resorption features, are precisely the demands for a good melanocyte graft. Bovine collagen I decellularized membrane scaffold fulfilled all of these requirements and provided a friendly niche for melanocytes derived from the ORS of hair follicles.

As one of the most putative skin protein constituents, collagen I represented a reliable adhesion surface for melanocyte grafts. Decellularized collagen scaffolds retained the geometry of the original extracellular matrix, providing 'cell-slots' that could be populated by epidermal cells. Such ready-to-wear skin architecture offered a niche for primary cell grafting that was very close to physiological terms [23] Furthermore, proven benevolent effects of collagen I on melanocytes strengthened the features of CCC membrane as melanocyte carrier.

In this study, we have already shown that the hair follicle ORSderived melanocytes successfully nested in the decellularized bovine collagen type I scaffold, protruded dendrites and interfaced with scaffold architecture, expressed melanocyte markers and produced melanin. The HM cells were able to populate the CCC membrane scaffold, they interfaced its inner surfaces, penetrated the depth of 100 $\mu \mathrm{m}$ (Figure 7) and their melanocyte features even exceeded those of the HM cells cultivated on adherent polystyrene surfaces. The HM proliferation rate on CCC membranes exceeded that of the HM in polystyrene-adherent culture by $28 \%$ (Figure 9); furthermore, the $\mathrm{HM}$ cultivated on CCC membranes produced $17 \%$ more melanin than polystyrene-adherent cells (Figure 10) which showed the advantages of three-dimensional cultivation and physiologically compatible surface. 
Citation: Savkovic V, Sülflow K, Rabe K, Schneider M, Simon JC (2012) Melanocytes from the Outer Root Sheath of Hair Follicles Cultivated on Collagen Membrane Improve their Melanotic Properties. J Tissue Sci Eng S11:004. doi:10.4172/2157-7552.S11-004

The enhanced melanotic function in the scaffolds in comparison to polystyrene adherent cultures speaks even more in favour of CCC membranes as a good support for melanocytes.

HM melanocytes retained their migratory capabilities on collagen I membranes, giving a fair chance of migration and re-distribution capability within the niche of native skin, after picking up the queue of their new in vivo post-graft environment.

Biodegradability and resorption capability of collagen I should enable optimal blending of such melanocyte graft into either epidermal or dermal tissue upon transplantation, without disturbing the organic architecture of the local matrix.

Both the promising $\mathrm{HM}$ cultivation improvements and the collagen I as graft constituent remain to be further explored in future. The cultivation procedure leaves room for acceleration in terms of differentiation of ORS stem cell and progenitor pool into melanocytes. Use of collagen I for melanocyte grafting offers prospects for a variety of upgrades in the direction of using human-derived scaffolds and introducing chemical modifications.

All of the above makes collagen I by all means a very decent candidate for the main component of melanocyte graft carriers.

\section{Acknowledgements}

The work presented here was made possible by funding from the German Federal Ministry of Education and Research (BMBF, PtJ-Bio, 0313909) and the German Research Council SFB TRR 67 project B3 to JCS. We would like to thank Prof. Thomas Magin for granting the access to the ApoTome set up and Dr. Wera Roth for introducing us to the ApoTome system basics.

\section{References}

1. Saleh HM, Salem SAM, El-Sheshetawy RS, Abd El-Samei AM (2008) Comparative Study of Psychiatric Morbidity and Quality of Life in Psoriasis, Vitiligo and Alopecia Areata. Egyptian Dermatology Online Journal 4: 2.

2. Porter J (2000) The psychological effects of vitiligo: response to impaired appearance. In: Vitiligo, a Monograph on the Basic and Clinical Science, Oxford 97-100.

3. Ongenae K, Van Geel N, De Schepper S, Naeyaert JM (2005) Effect of vitiligo on self-reported health-related quality of life. Br J Dermatol 152: 1165-1172.

4. Mattoo SK, Handa S, Kaur I, Gupta N, Malhotra R (2002) Psychiatric morbidity in vitiligo: prevalence and correlates in India. J Eur Acad Dermatol Venereo 16: $573-578$.

5. Sharma N, Koranne RV, Singh RK (2001) Psychiatric morbidity in psoriasis and vitiligo: a comparative study. J Dermatol 28: 419-423.

6. Gupta MA, Gupta AK (2003) Psychiatric and Psychological Co-Morbidity in Patients with Dermatologic Disorders: Epidemiology and Management. Am J Clin Dermatol 4: 833-842.

7. Sampogna F, Picardi A, Chren MM, Melchi CF, Pasquini P (2004) Association between poorer quality of life and psychiatric morbidity in patients with different dermatological conditions. Psychosom Med 66: 520-526.

8. Papadopoulos L, Bor R, Legg C (1999) Coping with the disfiguring effects of vitiligo: a preliminary investigation into the effects of cognitive-behavioural therapy. Br J Med Psychol 72: 385-396.

9. Yang YS, Cho HR, Ryou JH, Lee MH (2010) Clinical study of repigmentation patterns with either narrow-band ultraviolet B (NBUVB) or $308 \mathrm{~nm}$ excimer laser treatment in Korean vitiligo patients. Int J Dermatol 49: 317-323.

10. Casacci M, Thomas P, Pacifico A, Bonnevalle A, Paro Vidolin A, et al. (2007) Comparison between $308 \mathrm{~nm}$ monochromatic excimer light and narrowband UVB phototherapy (311-313 nm) in the treatment of vitiligo--a multicentre controlled study. J Eur Acad Dermatol Venereol 21: 956-963.

11. Forschner T, Buchholtz S, Stockfleth $E$ (2007) Current state of vitiligo therapy-evidence-based analysis of the literature. J Dtsch Dermatol Ges 5: 467-475.
12. Chen YF, Yang PY, Hu DN, Kuo FS, Hung CS, et al. (2004) Treatment of vitiligo by transplantation of cultured pure melanocyte suspension: analysis of 120 cases. J Am Acad Dermatol 51: 68-74.

13. Kim CY, Yoon TJ, Kim TH (2001) Epidermal grafting after chemical epilation in the treatment of vitiligo. Dermatol Surg 27: 855-856.

14. Lau K, Paus R, Tiede S, Day P, Bayat A (2009) Exploring the role of stem cells in cutaneous wound healing. Exp Dermatol 18: 921-933.

15. Rusfianti M, Wirohadidjodjo YW (2006) Dermatosurgical techniques for repigmentation of vitiligo. Int J Dermatol 45: 411-417.

16. Falabella R (2005) Surgical approaches for stable vitiligo. Dermatol Surg 31 1277-1284.

17. van Geel N, Ongenae K, De Mil M, Haeghen YV, Vervaet C, et al. (2004) Double-blind placebo-controlled study of autologous transplanted epidermal cell suspensions for repigmenting vitiligo. Arch Dermatol 140: 1203-1208.

18. Muleka SV (2005) Long-term follow-up study of 142 patients with vitiligo vulgaris treated by autologous, non-cultured melanocyte-keratinocyte cell transplantation. Int J Dermatol 44: 841-845.

19. Guerra L, Primavera G, Raskovic D, Pellegrini G, Golisano O, et al. (2003) Erbium: YAG laser and cultured epidermis in the surgical therapy of stable vitiligo. Arch Dermatol 139: 1303-1310.

20. Vanscheidt W, Hunziker T (2009) Repigmentation by outer-root-sheath-derived melanocytes: proof of concept in vitiligo and leucoderma. Dermatology 218 342-343.

21. Dieckmann C, Milkova L, Hunziker T, Emmendörffer A, Simon JC (2010) Human melanocytes can be isolated, propagated and expanded from plucked anagen hair follicles. Exp Dermatol 19: 543-545.

22. Savkovic V, Dieckmann C, Milkova L, Simon JC (2012) Improved method of differentiation, selection and amplification of human melanocytes from the hair follicle cell pool. Exp Dermatol 21: 948-950.

23. http://www.viscofan-bioengineering.com/wp-content/uploads/User-ProtocolCCC1.pdf.

24. http://www.promocell.com/fileadmin/promocell/PDF/C-39420.pdf.

25. Halaban R, Alfano FD (1984) Selective elimination of fibroblasts from cultures of normal human melanocytes. In vitro 20: 447-450.

26. Bar-Nun S, Shneyour Y, Beckmann JS (1983) G-418, an elongation inhibitor of $80 \mathrm{~S}$ ribosomes. Biochim Biophys Acta 741: 123-127.

27. http://rsb.info.nih.gov/ij.

28. Savkovic V, Gantzer H, Reiser U, Selig L, Gaiser S, et al. (2007) Clusterin is protective in pancreatitis through anti-apoptotic and anti-inflammatory properties. Biochem Biophys Res Commun 356: 431-437.

29. Biernaskie J (2010) Human hair follicles: "bulging" with neural crest-like stem cells. J Invest Dermatol 130: 1202-1204.

30. Tiede S, Kloepper JE, Bodò E, Tiwari S, Kruse C, et al. (2007) Hair follicle stem cells: walking the maze. Eur J Cell Biol 86: 355-376.

31. Yu H, Fang D, Kumar SM, Li L, Nguyen TK, et al. (2006) Isolation of a nove population of multipotent adult stem cells from human hair follicles. Am J Pathol 168: 1879-1888.

32. Hoffman RM (2006) The pluripotency of hair follicle stem cells. Cell Cycle 5 232-233.

33. Yu H, Kumar SM, Kossenkov AV, Showe L, Xu X (2010) Stem cells with neural crest characteristics derived from the bulge region of cultured human hair follicles. J Invest Dermatol 130: 1227-1236.

34. Amoh Y, Kanoh M, Niiyama S, Kawahara K, Sato Y, et al. (2009) Human and mouse hair follicles contain both multipotent and monopotent stem cells. Cell Cycle 8: 176-177.

35. Cotsarelis G, Sun TT, Lavker RM (1990) Label-retaining cells reside in the bulge area of pilosebaceous unit: implications for follicular stem cells, hair cycle, and skin carcinogenesis. Cell 61: 1329-1337.

36. Stenn KS, Paus R (2001) Controls of hair follicle cycling. Physiol Rev 81: 449 
Citation: Savkovic V, Sülflow K, Rabe K, Schneider M, Simon JC (2012) Melanocytes from the Outer Root Sheath of Hair Follicles Cultivated on Collagen Membrane Improve their Melanotic Properties. J Tissue Sci Eng S11:004. doi:10.4172/2157-7552.S11-004

37. Paus R, Foitzik K (2004) In search of the "hair cycle clock": a guided tour. Differentiation 72: 489-511.

38. Cotsarelis G, Sun TT, Lavker RM (1990) Label-retaining cells reside in the bulge area of pilosebaceous unit: implications for follicular stem cells, hair cycle, and skin carcinogenesis. Cell 61: 1329-1337.

39. Paus R, Cotsarelis G (1999) The biology of hair follicles. N Engl J Med 341: 491-497.

40. Stenn KS, Paus R (2001) Controls of hair follicle cycling. Physiol Rev 81: 449494

41. Cotsarelis G (2006) Epithelial stem cells: a folliculocentric view. J Invest Dermatol 126: 1459-1468.

42. Ito M, Liu Y, Yang Z, Nguyen J, Liang F, et al. (2005) Stem cells in the hair follicle bulge contribute to wound repair but not to homeostasis of the epidermis. Nat Med 11: 1351-1354.

43. Trantolo DJ, Lewandrows ki KU, Hasirci V, Altobelli DE, Wise DL (2003) Collagen: The Next Generation of Resorbable Biodevices in Surgery. Tissue Engineering and Novel Delivery Systems.

44. Fleck S, Simman R (2010) Modern Collagen Wound Dressings: Function and Purpose. The Journal of the American College of Certified Wound Specialists 2: $50-54$.
45. Supp DM, Boyce ST (2005) Engineered skin substitutes: practices and potentials. Clin Dermatol 23: 403-412.

46. Horch RE, Debus M, Wagner G, Stark GB (2000) Stark Cultured human keratinocytes on type I collagen membranes to reconstitute the epidermis. Tissue Eng 6: 53-67.

47. Liu J, Bian Z, Kuijpers-Jagtman AM, Von den Hoff JW (2010) Skin and ora mucosa equivalents: construction and performance. Orthod Craniofac Res 13 $11-20$.

48. Johnen C, Steffen I, Beichelt D, Bräutigam K, Witascheck T, et al. (2008) Culture of subconfluent human fibroblasts and keratinocytes using biodegradable transfer membranes. Burns 34: 655-663.

49. Lin SJ, Jee SH, Hsiao WC, Yu HS, Tsai TF, et al. (2006) Enhanced cell survival of melanocyte spheroids in serum starvation condition. Biomaterials 27: 1462 1469.

50. Swope VB, Supp AP, Schwemberger S, Babcock G, Boyce S (2006) Increased expression of integrins and decreased apoptosis correlate with increased melanocyte retention in cultured skin substitutes. Pigment Cell Res 19: 424 433.

51. Mulekar SV (2004) Long-term follow-up study of segmental and foca vitiligo treated by autologous, noncultured melanocyte-keratinocyte cell transplantation. Arch Dermatol 140: 1211-1215. 